\title{
Une brève histoire de l'adaptation : l'évolution conceptuelle au fil des rapports du GIEC (1990-2014)
}

\author{
Guillaume Simonet \\ Sciences de l'environnement, CDC Climat Recherche, pôle Politiques climatiques locales, 75009 Paris, France
}

\author{
Mots-clés : \\ adaptation ; \\ changements \\ climatiques ; \\ conceptualisation ; \\ barrière cognitive ; \\ GIEC
}

\section{Keywords:}

adaptation; climate

change;

conceptualization;

cognitive factor; IPCC

\begin{abstract}
Résumé - Les notions de vulnérabilité, de résilience et d'adaptation aux changements climatiques sont devenues centrales en même temps que sont de plus en plus étudiées les influences de leurs interprétations dans les prises de décision. La compréhension de l'adaptation évolue et un glissement d'une vision ajustement vers une perception transformationnelle s'observe. L'article analyse les avancées conceptuelles de l'adaptation dans les cinq rapports du Groupe intergouvernemental des experts sur l'évolution du climat de 1990 à 2014. Sa place prépondérante dans le dernier rapport (2014) reflète la difficulté d'en penser le concept, répond au besoin de faciliter son opérationnalisation et confirme sa pertinence pour appréhender la complexité sous-jacente à la problématique climatique. La poursuite de la réflexion sémantique contribue à la consolidation d'un champ de recherche prometteur et décloisonné.
\end{abstract}

\begin{abstract}
A brief history of adaptation: conceptual evolution across the IPCC reports (1990-2014). In the past decade, work on the concepts of vulnerability, resilience and adaptation became central in climate change literature. At the same time a growing number of studies have focussed on the influence of cognitive factors on decision-making processes, such as interpreting elusive concepts such as adaptation. Comprehension of adaptation has evolved considerably since its recognition as a response to climate change in the 90 s by the Intergovernmental Panel on Climate Change (IPCC). Currently protecting systems from weather events (the "adjustment adaptation" approach) is still the prevailing view in climate policies. Nevertheless, a shift toward a "transformation adaptation" approach is being observed. This position would take better account of the complexity of existing systems and allow reexamining their mechanisms (institutional, technical, financial). This paper aims to analyze conceptual advances on adaptation across the five IPCC reports from 1990 to 2014 . This contribution attempts to show that the prominence given to adaptation in the latest report (2014) reflects the cognitive difficulty of conceiving this concept, responds to the growing demand to facilitate its operationalization and confirms its relevance for a better understanding of the underlying complexity of climate change and the global environmental change issue. Pursuing reflection on the adaptation concept should certainly contribute to the emergence of a promising and interdisciplinary field of research.
\end{abstract}

\section{Introduction}

De plus en plus de recherches dans le domaine des sciences cognitives, en premier lieu en psychologie, se penchent sur l'étude des facteurs d'ordre cognitif à l'origine d'influences significatives sur la prise de décision dans le domaine des changements climatiques
(Gifford, 2008 ; Anderson, 2011). Parmi les facteurs psychologiques jouant sur les processus à l'origine des décisions, on retrouve la perception, les représentations, l'acquisition des connaissances, la mémoire, l'attitude, les émotions et l'interprétation des risques (Böhm et Pfister, 2000 ; Leiserowitz, 2006 ; Reser et al., 2011). La manière de percevoir l'environnement et ses risques, de le penser et d'interagir avec occupe une place prépondérante dans les

Auteur correspondant : guillaume.simonet@cdcclimat.com

GuillaumeSimonet est actuellement chercheur invité au laboratoire Géosciences Environnement(UMR5563) de l'Observatoire Midi-Pyrénées à Toulouse et en charge du projet de recherche ABSTRACT-colurba. 
articles traitant des obstacles cognitifs à la mise en place de réponses (Gifford, 2011). Ces travaux de recherche contribuent à une meilleure compréhension en matière d'impacts (directs et indirects) climatiques sur les systèmes (naturels et humains), à l'évolution des réflexions sur les concepts mobilisés (résilience, vulnérabilité, adaptation) ainsi qu'à l'amélioration des connaissances concernant les freins et leviers guidant les processus de décision en amont de la mise en œuvre d'actions (IPCC, 2014 ; Eyzaguirre et al., 2014).

$\mathrm{Au}$ cours de la dernière décennie, les réflexions portant sur la notion de vulnérabilité (physique et sociale) face aux enjeux des changements climatiques sont devenues centrales dans la littérature scientifique spécialisée (Oppenheimer et al., 2014 ; Quenault et al., 2011 ; Becerra et Peltier, 2009). Ces dernières années ont également vu un nouvel engouement pour le concept de résilience, perceptible notamment dans le renouvellement des dispositifs de gestion de risques, en partie parce qu'il est perçu comme prometteur pour faire face à des situations complexes et incertaines (Quenault, 2013). Concernant le concept d'adaptation, il affiche une popularité florissante, notamment depuis 2007 (Eyzaguirre et al., 2014), certains y voyant l'émergence d'un champ de recherche à part entière (Patt, 2013; Simonet, 2014a). Ces trois notions continuent d'évoluer dans leurs acceptions théoriques, de manière séparée ou associée (Vogel et al., 2007 ; Nelson et al., 2007), s'influençant parfois les unes les autres dans leur manière d'être pensées (Basset et Fogelman, 2013). Toutefois, la difficulté de traduire ces avancées en stratégies opérationnelles est régulièrement soulevée.

Ainsi, concernant le concept d'adaptation, plusieurs auteurs montrent invariablement que la signification du terme reste « fuyante » et varie selon les chercheurs, laissant une large marge d'interprétation (Smit et al., 2000 ; Füssel, 2007; Pelling, 2011 ; Mustelin et al., 2013). Au niveau local, les acteurs territoriaux et les décideurs publics aux prises avec la mise en œuvre des volets «adaptation» des différentes politiques climatiques se heurtent également à l'imprécision du terme (Adger et Barnett, 2009 ; Simonet, 2013a). Parmi les causes expliquant les origines de cette profusion interprétative, l'hypothèse de la complexité conceptuelle du terme issue de son historique sémantique et théorique est avancée. En effet, prisonnière de son étymologie en référence à la fois à une action (processus) et à la finalité (état) qui en résulte, l'adaptation engendre une ambivalence ouvrant à de multiples interprétations, notamment lorsqu'il s'agit de la traduire en actions pratiques (Simonet, 2009 ; Simonet, 2014b).

Cette difficulté cognitive n'a pas empêché la diffusion d'une pléthore de définitions de l'adaptation aux changements climatiques, parmi lesquelles celles en provenance des publications du Groupe intergouvernemental des experts sur l'évolution du climat (GIEC). La compréhension de l'adaptation a toutefois évolué depuis sa reconnaissance initiale comme une réponse aux changements climatiques dans les années 1990 (Eyzaguirre et al., 2014). Les premières définitions de l'adaptation du GIEC furent basées sur l'ajustement des systèmes aux impacts climatiques, issues des travaux sur la gestion des catastrophes naturelles. L'influence de cette posture, présente dès le second rapport (1995), fut manifeste: plus de $70 \%$ des articles de la littérature scientifique spécialisée mettent en avant cette dimension dès lors que l'adaptation est traitée (Basset et Fogelman, 2013). Aujourd'hui, c'est également l'idée de protection et d'ajustement aux aléas météorologiques qui prime dans la majorité des politiques publiques climatiques, bien que la confusion règne dès lors que les acteurs territoriaux sont interrogés sur une définition plus précise de ce que représente une "adaptation aux changements climatiques » (Simonet, 2011b).

Parallèlement à cette tendance, ces dernières années ont vu s'accroître le spectre interprétatif de ce que sousentend l'adaptation dans un contexte de changements climatiques. De cet exercice, la dimension «processus » émerge des réflexions, à travers notamment le concept de «transformation ». Reprenant les trois types d'adaptation caractérisés par Pelling (2011), les interprétations de l'adaptation trouvées dans la littérature spécialisée se classifient en trois tendances principales: « l'adaptation ajustée », «l'adaptation réformiste » et "l'adaptation transformative » (Basset et Fogelman, 2013). Alors que la première établit les impacts climatiques comme la source principale de la vulnérabilité des systèmes, la dernière met l'emphase sur l'importance de l'évolution des dimensions socioéconomiques et environnementales qui caractérisent les systèmes dans sa vulnérabilité face aux changements climatiques. La deuxième tendance se situe entre les deux. Ainsi, les travaux portant sur l'aspect «transformation » de l'adaptation résultent d'un nouveau domaine de recherche, dont les définitions et les interprétations continuent d'évoluer (O'Brien, 2012 ; Rickards et Howden, 2012 ; Park et al., 2012 ; Eyzaguirre et al., 2014).

L'influence de ces recherches s'est révélée lors du cinquième rapport (2014) du GIEC dans lequel l'adaptation accapare une place thématique plus importante que lors des derniers. En effet, marqué par une douzaine de nouveaux termes associés à l'adaptation dans son glossaire, c'est l'aspect « transformation » qui caractérise plus précisément le traitement consacré à l'adaptation dans ce dernier rapport, désormais en rupture avec les précédents. Dès lors, le passage de la vision «ajustement » vers une perception plus dynamique de l'adaptation, appelée «adaptation transformationnelle», marque un tournant dans l'interprétation de l'adaptation aux changements climatiques et la diffusion de sa signification. 
Tableau 1. Évolution des intitulés des différents tomes des rapports du GIEC.

\begin{tabular}{|l|l|l|l|}
\hline \multicolumn{1}{|c|}{ Rapport } & \multicolumn{1}{|c|}{ Working Group I } & \multicolumn{1}{c|}{ Working Group II } & \multicolumn{1}{c|}{ Working Group III } \\
\hline I (1990) & $\begin{array}{l}\text { Scientific Assessment of Climate } \\
\text { Change }\end{array}$ & Impacts Assessment of Climate Change & The IPCC Response Strategies \\
\hline II (1995) & The Science of Climate Change & $\begin{array}{l}\text { Impacts, Adaptations and Mitigation of } \\
\text { Climate Change: Scientific-Technical } \\
\text { Analyses }\end{array}$ & $\begin{array}{l}\text { Economic and Social } \\
\text { Dimensions of Climate } \\
\text { Change }\end{array}$ \\
\hline III (2001) & The Scientific Basis & Impacts, Adaptation and Vulnerability & Mitigation \\
\hline IV (2007) & The Physical Science Basis & Impacts, Adaptation and Vulnerability & Mitigation of Climate Change \\
\hline V (2014) & The Physical Science Basis & Impacts, Adaptation and Vulnerability & Mitigation of Climate Change \\
\hline
\end{tabular}

En effet, l'introduction d'un aspect plus dynamique permet de mieux prendre en compte la complexité des systèmes et ouvre la porte à la remise en question des pertinences des mécanismes (institutionnels, techniques, financiers) sous-jacents aux systèmes en place.

L'objectif de l'article est d'analyser la place de l'adaptation dans le cinquième rapport (2014) du GIEC ${ }^{1}$. À travers une comparaison de la place de l'adaptation au sein des cinq rapports du GIEC de 1990 à 2014, l'article interroge l'efficacité de l'élargissement sémantique observé quant à une meilleure interprétation de ce que représente "s'adapter aux changements climatiques». En quoi, les nouvelles définitions de l'adaptation peuvent faciliter sa mise en œuvre? L' « adaptation transformationnelle ", introduite dans ce dernier rapport, apporte-telle un éclairage nouveau ? Sommes-nous capables de penser l'adaptation aux changements climatiques ou avons-nous atteint une limite dans sa compréhension?

L'hypothèse défendue est que l'extension du spectre des définitions entourant l'adaptation confirme que la compréhension du terme reste problématique en soi, amenant à une impasse conceptuelle difficile à surmonter. En dépit de cela, l'auteur reste convaincu que le concept d'adaptation est une paire de lunettes appropriée pour mieux appréhender la complexité sousjacente à la problématique climatique, elle-même inscrite dans un contexte de changement global. La poursuite de la réflexion sémantique participe au réglage de la focale et de ce fait contribue à la consolidation d'un champ de recherche prometteur, notamment par l'interdisciplinarité qu'il exige.

\footnotetext{
1 Cet article a été réalisé dans le cadre du projet ABSTRACTcolurba qui a bénéficié de l'aide financière de l'Agence de l'environnement et de la maîtrise de l'énergie (Ademe) et de l'Agence française de développement (AFD) ainsi que du partenariat actif de l'Observatoire national sur les effets du réchauffement climatique (Onerc) et du Commissariat général à l'égalité des territoires (CGET).
}

\section{L'évolution de l'adaptation dans les cinq rapports du GIEC}

\section{Des intitulés qui se stabilisent dès le troisième rapport}

Publié environ tous les six ans, le rapport du GIEC a évolué depuis sa première version (1990), tant dans son contenu, qu'en ce qui concerne le nombre de scientifiques associés et la diversité disciplinaire des travaux pris en compte pour étayer l'état du système climatique à l'échelle globale. Ainsi, au fur et à mesure des rapports se sont précisées les observations, la confirmation des origines anthropiques du phénomène, la nature des impacts et les pistes de solutions. De ce fait, la structure des documents publiés a elle aussi progressé, l'intitulé des titres révélant les thématiques abordées dans chacun des «Working Group » ${ }^{2}$ responsables des trois tomes qui composent un rapport (Tab. 1).

La notion d'adaptation vit essentiellement le jour dans le deuxième rapport du GIEC (1995) à l'image de son apparition dans l'intitulé du second tome. Ainsi, bien que l'adaptation fît partie du premier rapport (1990) dans la sémantique textuelle du document, notamment dans le troisième tome, ce volet restait en marge dans le paysage conceptuel, scientifique et politique de l'époque, pensant que la technologie ou la capacité historique de s'adapter au climat suffisait à régler la problématique climatique ${ }^{3}$. Lors de son élaboration, quatre sous-groupes de travail furent créés pour travailler sur les stratégies de réponses face aux changements climatiques, les deux premiers sur la réduction des émissions

\footnotetext{
2 Afin d'accroître la cohérence dans l'exercice de comparaison des rapports du GIEC entre 1990 et 2014, l'auteur fait le choix de garder les titres, intitulés ou termes en anglais.

3 «While noting the need for adaptive response strategies, it should also be recognized that adaptation to climate is at least as old as the human species » (IPCC, 1990, tome 3, p. 174).
} 
de gaz à effet de serre (GES) et les deux derniers sur l'adaptation aux impacts à travers les questions de gestion des zones côtières et de l'utilisation des ressources naturelles ${ }^{4}$, alimentés par les quelques recherches de terrain disponibles (Butzer, 1980 ; Rosenberg et al., 1989; Jodha, 1989). Auréolée par le succès du protocole de Montréal (1987) relatif aux substances appauvrissant la couche d'ozone, la communauté internationale pensait rapidement régler la question émergente des changements climatiques en dirigeant les efforts à fournir sur ses causes, laissant de côté la question des conséquences d'une problématique aux tendances et aux impacts encore hypothétiques. En somme, les changements climatiques se résumaient à une question d'ajustement technique et prescriptif visant à réduire les émissions de GES.

À l'instar de sa terminologie plurielle "Adaptations » dans l'intitulé du second tome du deuxième rapport (1995), l'adaptation fut d'abord appréhendée à travers les options techniques à mettre en place, dispersées selon les secteurs et les thématiques abordées. Seul le chapitre 18 portant sur la santé aborda les liens entre adaptation et changements socioéconomiques (section 18-5 «Options for Adaptation», p. 579), introduisant l'idée de « transformation » qui allait progressivement s'imposer dans les rapports suivants (Basset et Fogelman, 2013). Mis à part cette section, les idées véhiculées sur la notion d'adaptation face aux impacts climatiques s'inspirèrent directement des travaux de recherche sur la gestion des catastrophes naturelles. Cette influence eut pour effet de mettre l'accent uniquement sur la partie « ajustement » du terme, en établissant un lien direct entre adaptation et impacts. Cette posture fut confortée par l'accumulation d'impacts climatiques aux dégâts matériels et humains croissants dans les pays insulaires et les pays en développement, lesquels réussirent peu à peu à attirer l'attention sur la nécessité de l'adaptation au sein des négociations internationales (Schipper, 2006). Cette influence, ajoutée au constat grandissant de l'inefficacité des politiques de réduction des émissions de GES et d'une volonté marquée de maintenir une croissance économique vigoureuse à partir de ressources énergétiques carbonées, permit à l'adaptation de prendre son envol (Marquet et Salles, 2014). L'essor de l'intérêt pour l'adaptation gagna

\footnotetext{
4 «The four RSWG Subgroups were tasked with developing a range of climate change response strategies in the areas of: (1) Energy and Industry; (2) Agriculture and Forestry; (3) Coastal Zone Management; and (4) Resource Use and Management. It was agreed that the first two subgroups would consider measures for limiting net greenhouse gas emissions from the energy, industry, agriculture, and forestry sectors, and that the latter two subgroups would deal with measures for adapting to the impacts of climate change on coastal regions and natural resources » (IPCC, 1990, tome 3, p. 65).
}

les instances internationales jusqu'à l'échelle des collectivités territoriales au travers de la multiplication des politiques publiques et des outils (Richard, 2014).

L'adaptation perdit son pluriel dans l'intitulé du second tome dès le troisième rapport (2001) pour être associée à "Vulnerability », formant avec "Impacts » un triptyque immuable. Le passage au singulier de la notion est loin d'être anecdotique. En premier lieu, il dénote l'abandon progressif d'une vision de l'adaptation principalement axée sur des mesures techniques visant à protéger les systèmes des impacts climatiques. Ensuite, cette modification coïncide avec l'arrivée de la vulnérabilité comme concept central des réflexions (Burton et al., 2002) et la prise en compte croissante des dimensions socioéconomiques, jusque-là cantonnées dans le troisième tome du deuxième rapport (1995). Ainsi, le second tome du rapport du GIEC devint dès 2001 celui dédié à la gestion des conséquences des changements climatiques. Ce choix provoqua la migration de la mitigation vers le troisième tome, dès lors consacré aux actions s'attaquant aux émissions de GES d'origine anthropique.

Concernant le premier tome des rapports du GIEC, sa vocation à traiter des aspects climatologiques n'a pas varié au fil du temps. Néanmoins, l'introduction dans son intitulé du terme "Physical » en 2007 à la place de "Scientific» indiqua une influence croissante des sciences humaines et sociales dans l'exercice scientifique attenant à la problématique climatique d'origine anthropique, auparavant accaparée par les sciences du climat. Cette précision terminologique souligna ainsi l'importance accrue accordée aux dimensions autres que celles physiques dans l'étude des changements climatiques. De ce fait, elle envoya un signal fort à la communauté scientifique internationale quant à la volonté de mettre fin à l'association de certaines disciplines au terme "Science » au détriment d'autres. Enfin, cette évolution symbolisa la volonté d'aborder la problématique climatique de manière systémique, c'est-à-dire dans toute sa complexité d'échelles, de temps et d'interrelations entre les systèmes de toutes sortes. Ainsi, concrétisant cet élargissement disciplinaire, le cinquième rapport présente pour la première fois un paragraphe intitulé "Psychology " dans le chapitre 2 "Foundations for Decisionmaking ", abordant l'influence des facteurs cognitifs (perception, émotions ou encore compréhension du risque) sur la prise de décision.

\section{Des sommaires qui grossissent à la faveur de chapitres sur l'adaptation}

L'analyse des sommaires des rapports du GIEC portant sur l'adaptation donne une idée de sa place dans les réflexions. Le premier rapport (1990) du GIEC fut structuré différemment puisque l'adaptation fut abordée à la fois dans le tome 2 et dans le tome 3 (Tab. 2). Dès lors, la 
Tableau 2. Sommaire du deuxième et troisième tome du premier rapport du GIEC (1990).

\begin{tabular}{|c|c|c|}
\hline Rapport & \multicolumn{2}{|c|}{ Sommaire (par chapitres) } \\
\hline II (1990) & $\begin{array}{l}\text { Working Group II: Impacts Assessment of Climate } \\
\text { Change } \\
\text { I - Scenarios Used in the Report } \\
\text { II - Agriculture and Forestry } \\
\text { III - Natural Terrestrial Ecosystem } \\
\text { IV - Human Settlement; the Energy, Transport and } \\
\text { Industrial Sectors; Human Health; Air Quality and } \\
\text { Changes in Ultraviolet B Radiation } \\
\text { VI - Oceans and Coastal Zones } \\
\text { VII - Seasonal Snow Cover, Ice and Permafrost }\end{array}$ & $\begin{array}{l}\text { Working Group III: The IPCC Response Strategies } \\
\text { I Policymakers Summary } \\
\text { II IPCC Response Strategies Working Group Reports } \\
\text { 1. Introduction } \\
\text { 2. Emissions Scenarios } \\
\text { Subgroup Reports } \\
\text { 3. Energy and Industry } \\
\text { 4. Agriculture, Forestry, and Other Human Activities } \\
\text { 5. Coastal Zone Management } \\
\text { 6. Resource Use and Management } \\
\text { Implementation Measures } \\
\text { 7. Public Education and Information } \\
\text { 8. Technology Development and Transfer } \\
\text { 9. Economic (Market) Measures } \\
\text { 10. Financial Mechanisms } \\
\text { 11. Legal and Institutional Mechanisms }\end{array}$ \\
\hline
\end{tabular}

pertinence de l'analyse porte davantage sur les seconds tomes des rapports traitant de la notion d'adaptation (les quatre derniers) [Tab 3]. Lors du second rapport (1995), l'adaptation fut abordée en termes d'options en même temps que les impacts de manière transversale au sein de chapitres séparés selon les secteurs d'activité, les ressources naturelles et les écosystèmes.

Le troisième rapport (2001) introduisit des chapitres focalisant sur les régions géographiques, fusionnant plusieurs chapitres traitant de secteurs d'activité ou de ressources naturelles. L'adaptation composa également le chapitre 1 introductif et le chapitre 18, abordée en relation avec le développement durable, notion en plein essor à la veille d'un sommet de Johannesburg (2002) devant marquer les dix ans du sommet de la Terre de Rio (1992).

Dans le quatrième rapport (2007), la notion d'adaptation accapara le chapitre 17 en abordant notamment son opérationnalisation, les contraintes à l'action et les limites. Le chapitre suivant posa les jalons de passerelles conceptuelles et de pratiques entre l'adaptation et la mitigation dans une démarche intégrée de lutte contre les changements climatiques. Ce fait marqua un tournant : compte tenu de l'inertie des émissions de GES passées, de celles présentes et futures, et du fait de la conjoncture socioéconomique et démographique mondiale, l'adaptation fut désormais perçue comme inéluctable. Poursuivant cette prise de conscience à l'échelle de la communauté internationale, les efforts (financiers, techniques, politiques) englobèrent ceux portant sur les conséquences des changements climatiques et non plus seulement sur les causes. C'est dans cette dynamique que la vulnérabilité prit un nouvel essor conceptuel (Füssel, 2007).
Dans cette lignée, le cinquième rapport (2014) poursuit l'importance croissante donnée à l'adaptation. En effet, pas moins de quatre chapitres lui sont entièrement consacrés au sein d'une partie intitulée comme telle, en plus de chapitres sur ses relations avec le développement durable, la mitigation et un nouveau terme: "Climate-Resilient Pathways".

\section{Une définition qui évolue timidement}

Outre les intitulés et les sommaires des seconds tomes des rapports du GIEC, l'analyse de leur glossaire permet d'avoir une idée sur l'évolution interprétative de la notion d'adaptation à travers la comparaison des définitions qui y sont présentées (Tab. 4).

L'utilisation d'un glossaire regroupant les définitions des termes employés débuta au deuxième rapport (1995), le premier (1990) ne présentant qu'une succincte liste d'acronymes. Dans cette première version de glossaire, le terme "Adaptability» fut préféré à celui $\mathrm{d}^{\prime}$ «Adaptation». Toutefois, une définition de l'adaptation fut présentée dans la partie IV «Technical Appendices ", chapitre 26 , section $26.8^{6}$ dans laquelle l'idée

5 Les parties « Part I - Introductory Materials », « Part III Assessment of Mitigation Options » et « Part IV - Technical Appendices » n'ont pas été incorporées dans ce tableau.

6 "Adaptation is concerned with responses to both the adverse and positive effects of climate change. It refers to any adjustment - whether passive, reactive, or anticipatory - that can respond to anticipated or actual consequences associated with climate change. It thus implicitly recognizes that future climate changes will occur and must be accommodated in policy » (IPCC, 1995, tome 2, p. 831). 
Tableau 3. Comparaison des sommaires des seconds tomes entre 1995 et $2014^{6}$.

\begin{tabular}{|c|c|c|}
\hline Rapport & \multicolumn{2}{|c|}{ Sommaire (par chapitre) } \\
\hline II (1995) & $\begin{array}{l}\text { Part II-Assessment of Impacts and Adaptation } \\
\text { options } \\
\text { 1. Climate Change Impacts on Forests } \\
\text { 2. Rangelands in a Changing Climate: Impacts, } \\
\text { Adaptation and Mitigation } \\
\text { 3. Deserts in a Changing Climate: Impacts } \\
\text { 4. Land Degradation and Desertification } \\
\text { 5. Impacts of Climate Change on Mountain } \\
\text { Regions } \\
\text { 6. Non-Tidal Wetlands } \\
\text { 7. The Cryosphere: Changes and Their Impacts } \\
\text { 8. Oceans } \\
\text { 9. Coastal Zones and Small Islands } \\
\text { 10. Hydrology and Freshwater Ecology }\end{array}$ & $\begin{array}{l}\text { 11. Industry, Energy, and Transportation: Impacts and } \\
\text { Adaptation } \\
\text { 12. Human Settlements in a Changing Climate: Impacts and } \\
\text { Adaptation } \\
\text { 13. Agriculture in a Changing Climate: Impacts and Adaptation } \\
\text { 14. Water Resources Management } \\
\text { 15. Wood Production under Changing Climate and Land Use } \\
\text { 16. Fisheries } \\
\text { 17. Financial Services } \\
\text { 18. Human Population Health }\end{array}$ \\
\hline III (2001) & $\begin{array}{l}\text { 1. Overview of Impacts, Adaptation, and Vulnera- } \\
\text { bility to Climate Change } \\
\text { 2. Methods and Tools } \\
\text { 3. Developing and Applying Scenarios } \\
\text { 4. Hydrology and Water Resources } \\
\text { 5. Ecosystems and Their Goods and Services } \\
\text { 6. Coastal Zones and Marine Ecosystems } \\
\text { 7. Human Settlements, Energy, and Industry } \\
\text { 8. Insurance and Other Financial Services } \\
\text { 9. Human Health }\end{array}$ & $\begin{array}{l}\text { 10. Africa } \\
\text { 11. Asia } \\
\text { 12. Australia and New Zealand } \\
\text { 13. Europe } \\
\text { 14. Latin America } \\
\text { 15. North America } \\
\text { 16. Polar Regions (Arctic and Antarctic) } \\
\text { 17. Small Island States } \\
\text { 18. Adaptation to Climate Change in the Context of Sustainable } \\
\text { Development and Equity } \\
\text { 19. Vulnerability to Climate Change and Reasons for Concern: A } \\
\text { Synthesis }\end{array}$ \\
\hline IV (2007) & $\begin{array}{l}\text { 1. Assessment of Observed Changes } \\
\text { and Responses in Natural and Managed Systems } \\
\text { 2. New Assessment Methods and the Characteri- } \\
\text { zation of Future Conditions } \\
\text { 3. Freshwater Resources and Their Management } \\
\text { 4. Ecosystem, Their Properties, Goods and Services } \\
\text { 5. Food, Fibre and Forest Products } \\
\text { 6. Coastal Systems and Low-Lying Areas } \\
\text { 7. Industry, Settlement and Society } \\
\text { 8. Human Health }\end{array}$ & $\begin{array}{l}\text { 9. Africa } \\
\text { 10. Asia } \\
\text { 11. Australia and New Zealand } \\
\text { 12. Europe } \\
\text { 13. Latin America } \\
\text { 14. North America } \\
\text { 15. Polar Regions (Arctic and Antarctic) } \\
\text { 16. Small islands } \\
\text { 17. Assessment of adaptation practices, options, constraints and } \\
\text { capacity } \\
\text { 18. Inter-relationships between adaptation and mitigation } \\
\text { 19. Assessing key vulnerabilities and the risk from climate change } \\
\text { 20. Perspectives on climate change and sustainability }\end{array}$ \\
\hline V (2014) & $\begin{array}{l}\text { PART A: GLOBAL AND SECTORAL ASPECTS } \\
\text { Context for the AR5 } \\
\text { 1. Point of Departure } \\
\text { 2. Foundations for Decision-Making } \\
\text { Natural and Managed Resources and Systems, and Their } \\
\text { Uses } \\
\text { 3. Freshwater Resources } \\
\text { 4. Terrestrial and Inland Water Systems } \\
\text { 5. Coastal Systems and Low-Lying Areas } \\
\text { 6. Ocean Systems } \\
\text { 7. Food Security and Food Production Systems } \\
\text { Human Settlements, Industry, and Infrastructures } \\
\text { 8. Urban Areas } \\
\text { 9. Rural Areas } \\
\text { 10. Key Economic Sectors and Services } \\
\text { Human Health, Well-Being, and Security } \\
\text { 11. Human Health: Impacts, Adaptation, } \\
\text { and Co-benefits } \\
\text { 12. Human Security } \\
\text { 13. Livelihoods and Poverty }\end{array}$ & $\begin{array}{l}\text { Adaptation } \\
\text { 14. Adaptation needs and options } \\
\text { 15. Adaptation planning and implementation } \\
\text { 16. Adaptation opportunities, constraints, and limits } \\
\text { 17. Economics of adaptation } \\
\text { Multi-Sector Impacts, Risks, Vulnerabilities, and Opportunities } \\
\text { 18. Detection and attribution of observed impacts } \\
\text { 19. Emergent risks and key vulnerabilities } \\
\text { 20. Climate-resilient pathways: adaptation, mitigation and sus- } \\
\text { tainable development } \\
\text { PART B: REGIONAL ASPECTS } \\
\text { 21. Regional context } \\
\text { Regional Chapters } \\
\text { 22. Africa } \\
\text { 23. Europe } \\
\text { 24. Asia } \\
\text { 25. Australasia } \\
\text { 26. North America } \\
\text { 27. Central and South America } \\
\text { 28. Polar Regions } \\
\text { 29. Small Islands } \\
\text { 30. The Ocean }\end{array}$ \\
\hline
\end{tabular}


Tableau 4. Évolution des définitions de l'adaptation dans les différents rapports du GIEC.

\begin{tabular}{|l|l|}
\hline Rapport & \multicolumn{1}{|c|}{ Définition de l'adaptation } \\
\hline I (1990) & Néant. \\
\hline $\begin{array}{l}\text { II (1995) } \\
\text { Glossaire }\end{array}$ & $\begin{array}{l}\text { Adaptability: the degree to which adjustments are possible in practices, processes, or structures of systems to pro- } \\
\text { jected or actual changes of climate; adaptation can be spontaneous or planned, and can be carried out in response to } \\
\text { or in anticipation of changes in conditions (IPCC, 1995, p. 863). }\end{array}$ \\
\hline $\begin{array}{l}\text { III (2001) } \\
\text { Glossaire }\end{array}$ & $\begin{array}{l}\text { Adjustment in natural or human systems in response to actual or expected climatic stimuli or their effects, which } \\
\text { moderates, harm or exploits beneficial opportunities. Various types of adaptation can be distinguished, including } \\
\text { anticipatory and reactive adaptation, private and public adaptation, and autonomous and planned adaptation } \\
\text { (IPCC, 2001, p. 982). }\end{array}$ \\
\hline $\begin{array}{l}\text { IV (2007) } \\
\text { Glossaire }\end{array}$ & $\begin{array}{l}\text { Adjustment in natural or human systems in response to actual or expected climatic stimuli or their effects, which } \\
\text { moderates harm or exploits beneficial opportunities. Various types of adaptation can be distinguished, including } \\
\text { anticipatory, autonomous and planned adaptation (IPCC, 2007, p. 869). }\end{array}$ \\
\hline $\begin{array}{l}\text { V (2014) } \\
\text { Glossaire }\end{array}$ & $\begin{array}{l}\text { The process of adjustment to actual or expected climate and its effects. In human systems, adaptation seeks to moder- } \\
\text { ate harm or exploit beneficial opportunities. In natural systems, human intervention may facilitate adjustment to } \\
\text { expected climate and its effects (IPCC, 2014). }\end{array}$ \\
\hline
\end{tabular}

d'ajustement prédomine dans une simple perspective de protéger les systèmes des impacts climatiques. C'est également dans cette partie davantage destinée aux praticiens qu'il est mentionné que " deux types de réponses [face aux changements climatiques] peuvent être identifiées : la mitigation et l'adaptation ». Cette affirmation est suivie d'un court paragraphe définissant les termes " mitigation» et "adaptation», marquant leur envol dans les années suivantes. En effet, force est de constater que le terme "mitigation " n'apparaît quasiment pas dans les textes des deuxièmes et troisièmes tomes ${ }^{7} \mathrm{du}$ premier rapport (1990), avant d'être intégré dans le glossaire du second rapport (1995).

La présentation de ces deux « réponses » pour lutter contre les changements climatiques, présentées de manière distincte et justifiées par le fait que l'une s'occupe des causes tandis que l'autre a pour mission de gérer les conséquences, est importante à souligner. En effet, cette nette séparation eut possiblement une influence non négligeable dans le monde institutionnel et scientifique, ayant pu entretenir le conflit entre les tenants de la mitigation et ceux promouvant l'adaptation. Représentés par l'opposition entre les pays riches (mitigation) et les pays pauvres (adaptation), par l'occultation temporaire de l'adaptation de la scène politique ou par une séparation des fonds, les termes n'ont eu de cesse de s'opposer malgré de nombreux tenants de leur intégration. Il faut attendre le quatrième rapport (2007) et le chapitre 19 «Interrelationships between adaptation and mitigation » pour stabiliser un chevauchement entre les deux notions, inévitable dès la phase d'opérationnalisation (Simonet, 2011b).

7 Le terme "mitigation » apparaît deux fois dans le tome 2 et quatre fois dans le tome $3 \mathrm{du}$ premier rapport (1990) du GIEC.
Dans le glossaire du troisième rapport (2001), « Adaptability» ne comporta plus de définition, renvoyé à «Adaptive Capacity», tandis qu'«Adaptation » y fit son entrée avec une définition distinguant les dimensions biophysiques de celles des systèmes humains. En mentionnant les dimensions socioéconomiques, le concept d'adaptation se rapprocha de celui de la vulnérabilité, détaillant davantage les éléments déterminants de la « capacité adaptative » (technologie, ressources économiques, information et aptitudes, infrastructures, institutions et équité) sur laquelle les actions d'adaptation peuvent se développer. Néanmoins, bien que présentée sous différentes formes (anticipatrice, réactive, publique, privée, autonome ou planifiée), l'adaptation restait principalement teintée $d^{\prime}$ «ajustement». Les liens entre la distribution des ressources, la pauvreté et les pouvoirs ne furent pas discutés et l'adaptation demeura confinée à une simple liste de conditions techniques pouvant être traitées sans remise en cause de l'ordre social et politique établi (Basset et Fogelman, 2013). Pourtant, une définition de l'adaptation distincte du glossaire fut établie au chapitre 18, introduisant l'idée de " processus $»^{8}$.

La définition de l'adaptation présentée dans le quatrième rapport (2007) reprit les mêmes termes que dans le rapport précédent (2001) et ne présenta pas de réelles modifications, excepté la disparition des références à l'adaptation réactive, publique et privée. Malgré l'introduction de chapitres sur les barrières et limites de l'implantation de mesures et sur les interrelations avec la

8 «Adjustments in ecological, social, or economic systems in response to actual or expected climatic stimuli and their effects or impacts. It refers to changes in processes, practices, and structures to moderate potential damages or to benefit from opportunities associated with climate change » (IPCC, 2001, tome 2, p. 879). 


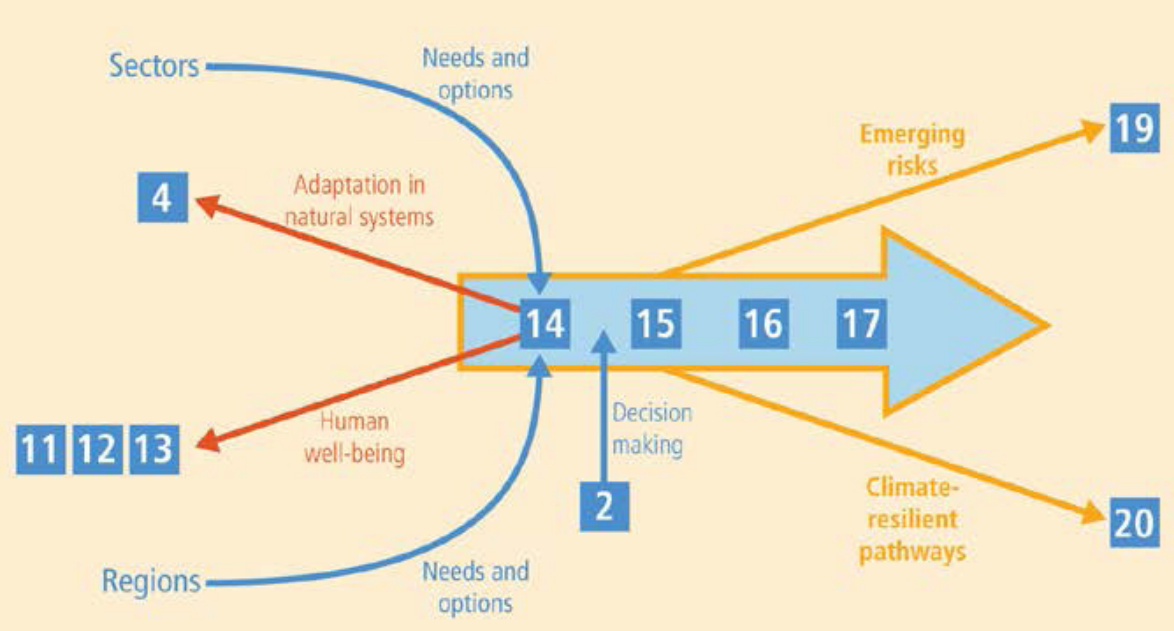

Fig. Liens entre les quatre chapitres de la partie « Adaptation» (14 à 17) et les autres chapitres (Figure 14-1 du chapitre 14, IPCC, 2014).

mitigation (chapitre 17 et chapitre 18), la notion d'adaptation fut encore largement décrite comme un ajustement visant à réduire la vulnérabilité des systèmes aux stimuli des changements climatiques. L'adaptation demeura confinée à un rôle de réponse palliative, occultant toujours la dimension relative à la «transformation » des systèmes, laquelle n'apparut pas comme une option envisageable (Basset et Fogelman, 2013).

Dans le cinquième et dernier rapport (2014), la définition se modifie subtilement via l'ajout de "seeks", laquelle introduit un élément de dynamique. Cet ajout rend la définition plus souple, qui ne va pas sans rappeler la signification de la traduction chinoise du terme « adaptation » à travers l'idéogramme (mandarin simplifié) 《适篮 》(shiying), lequel peut être défini comme «tenter de s'habituer à quelque chose de nouveau » (Simonet, 2008). Toutefois, bien que la définition de l'adaptation n'ait pas radicalement été modifiée, le glossaire du cinquième rapport présente une multitude de nouveaux termes attenants à celle-ci, plaçant la notion au cœur même de la réflexion.

\section{L'adaptation, colonne vertébrale du tome 2 du cinquième rapport}

Le cinquième rapport (2014) du GIEC marque une rupture avec ses précédents en consacrant une partie entière à l'adaptation, composée de quatre chapitres (14, 15,16 et 17), en plus de celui portant sur les relations conceptuelles avec le développement durable (20). L'adaptation est également abordée de manière centrale dans le chapitre 2 traitant de la prise de décision, de manière plus superficielle dans les chapitres $4,11,12$ et 13 et dans le chapitre portant sur les vulnérabilités (19). L'importance donnée à l'adaptation au sein de la structure du tome 2 est représentée à la première figure $\mathrm{du}$ chapitre 14 (Fig.). Ainsi, la disposition des chapitres sur l'adaptation (14 à 17) semble symboliquement traduire la volonté d'en constituer la colonne vertébrale ou le cœur du tome 2.

La justification de ce choix structurel est décrite dans le chapitre 15 (p. 4) où il est mentionné que l'importance des rapports scientifiques et de la couverture médiatique au sujet de l'adaptation aux changements climatiques a encouragé un nombre croissant d'actions dans les pays aussi bien développés qu'en développement, représentant un progrès majeur depuis le quatrième rapport. Ainsi, cette place consacrée à l'adaptation se concrétise dans le glossaire, dont la version 2014 présente une multitude de modifications par rapport aux précédents rapports (Tab. 5). Là encore, une note de bas de page associée à la définition du terme "Adaptation » précise qu'à l'image des avancées scientifiques, les entrées du glossaire diffèrent en termes de longueur et de focus par rapport aux précédents glossaires. Concrètement, douze nouveaux termes référant directement à la notion $\mathrm{d}^{\prime}$ adaptation ${ }^{9}$ font leur apparition dans le glossaire de 2014 par rapport à celui de 2007, tandis que quatre termes en disparaissent. Au niveau des termes dont la définition se réfère explicitement à la notion d'adaptation $^{10}$, six termes apparaissent à la faveur de trois qui disparaissent.

\footnotetext{
9 Recherche effectuée dans le glossaire des termes possédant la racine « adapt* .

10 Termes dont la définition contenait au moins une fois la racine « adapt* $»$.
} 
Tableau 5. Évolution de la place de l'adaptation dans les glossaires du GIEC entre 1995 et 2014.

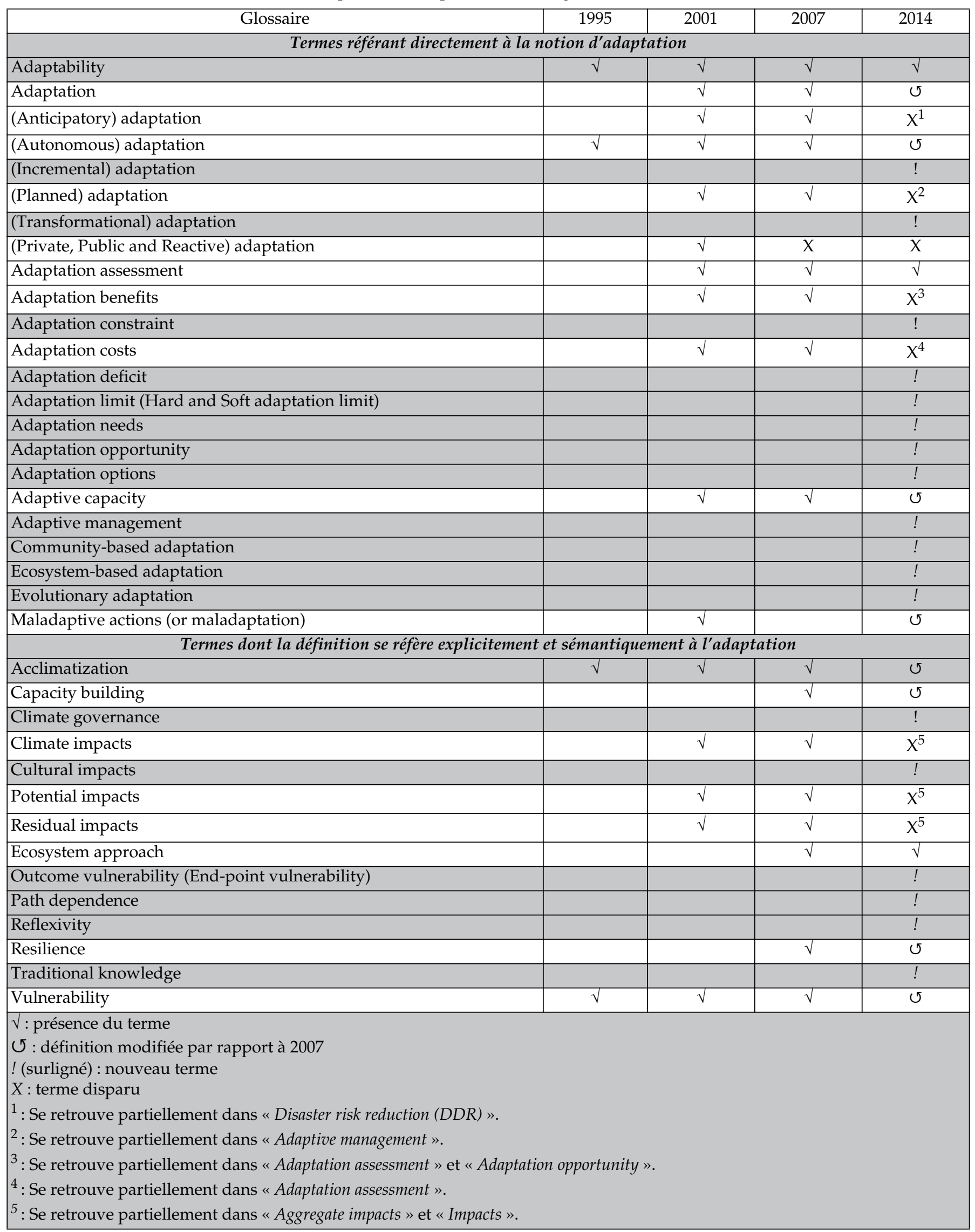




\section{De nouveaux termes se référant à l'adaptation... et à la mitigation !}

Les termes nouvellement présents dans le glossaire 2014 peuvent être différemment répartis. Tout d'abord, deux nouvelles notions accompagnent la définition sensu stricto de l'adaptation ("Incremental adaptation» et «Transformational adaptation»), apparaissant respectivement comme les nouvelles terminologies des dimensions «ajustement » et " processus » du concept d'adaptation. D'ailleurs, le texte suggère de se référer également à "Autonomous adaptation», «Evolutionary adaptation » et «Transformation». Ensuite, de nouveaux termes se réfèrent aux contraintes, limites, besoins, opportunités, options et au déficit de l'adaptation, apparaissant comme autant de précision praticienne ayant pour objectif de faciliter les contours de l'action à mettre en place, tout comme d'autres termes davantage reliés à des pratiques ("Community-based adaptation», «Ecosystem-based adaptation», «Adaptive management»). Enfin, le glossaire 2014 réintègre la notion de "Maladaptation", apparue dans le troisième rapport (2001) puis disparue lors du quatrième (2007), laissant supposer l'importance de cas observés d'actions contreproductives. Au-delà des termes se référant directement à l'adaptation, il y a ceux qui se réfèrent à l'adaptation dans leur définition, parmi lesquels «Climate governance », «Cultural impacts » et "Traditional knowledge », élargissant le cadre d'action et les domaines d'intervention reliés aux stratégies d'adaptation.

Au-delà de cette nouvelle terminologie de l'adaptation, le glossaire 2014 se pose également en rupture avec la terminologie attenante à la mitigation, présente sous deux définitions, une spécifiquement dédiée aux changements climatiques («Mitigation [of climate change] ») et l'autre aux catastrophes naturelles et à la notion de « risque » (« Mitigation [of disaster and disaster] »). Cette déclinaison accentue la confusion terminologique générale et rejoint l'ambigüité sémantique déjà soulevée entre « atténuation » et « adaptation » dans la traduction française du glossaire du quatrième rapport (2007) [Simonet, 2011a, p. 415].

\section{De nouvelles définitions pour plus de précisions?}

Dès lors, la question se pose quant à l'intérêt de préciser la définition de l'adaptation, ce que le chapitre 14 (p. 853) du cinquième rapport (2014) anticipe : "Why do the precise definitions about adaptation activities matter? » Argumenté d'une vingtaine de lignes, le premier point de la réponse met en avant la rapidité observée des changements climatiques et, désormais, son inévitable prise en compte dans les délibérations et décisions à toutes échelles d'organisation, de la communauté au parlement. Après une série d'exemples de questions plutôt orientées vers des pistes de réponses fournies dans le reste du rapport, il est ensuite précisé qu'une définition de l'adaptation permet d'établir un cadre de travail pour discuter des manières $d^{\prime}$ aborder les nouveaux défis et d'en préciser leurs portées, à défaut de prétendre répondre à toutes ces questions.

Le second point mentionne l'aspect «politique». Ainsi, à l'image de l'importance désormais actée de l'adaptation dans les négociations internationales, le texte évoque l'acceptation par les pays développés de soutenir les coûts d'adaptation des pays en développement via de nouvelles ressources financières, citant l'accord de Cancún (2010) et le montant envisagé du fonds dédié (10 milliards de dollars par an). Il est ensuite affirmé que l'adaptation est mieux réalisée une fois intégrée à de larges objectifs de planification territoriale déjà en œuvre, sous-entendant le «mainstreaming». Les exemples pris pour illustrer cet argument sont l'amélioration de la répartition de l'accès à l'eau et de la fiabilité des systèmes de transport.

Enfin, signalant qu'il est difficile de distinguer le coût de la remise en état d'une infrastructure endommagée par de mauvaises conditions climatiques de celui d'une adaptation aux changements climatiques, l'argumentaire termine sur l'idée que l'adaptation constitue avant tout une négociation. En effet, arguant de la difficulté d'établir des réponses précises, les auteurs mentionnent que «plus nous sommes d'accord sur ce que constitue l'adaptation, et plus il sera facile de parvenir à des accords réalisables ${ }^{11} »$.

\section{Une sémantique au gré des influences ?}

Les points soulignés dans l'argumentaire interrogent. Tout d'abord, l'importance d'être «d'accord» sur ce qu'elle « constitue » témoigne qu'en dehors du glossaire et de sa sémantique, l'adaptation aux changements climatiques se définit (avant tout ?) à travers le croisement d'interprétations. Ainsi, que «constitue» l'adaptation aux yeux des acteurs en place ? Pour les organismes prestataires de services, l'adaptation aux changements climatiques représente un marché potentiel émergent notamment dans les domaines de l'énergie et de l'eau (Simonet, 2011a). Ainsi, les intérêts se retrouvent dans les investissements et les services que le secteur privé peut offrir aux collectivités territoriales pour les aider à

\footnotetext{
11 «A precise answer may never be possible but the closer we agree as to what constitutes adaptation, the easier it will be to come to workable agreements » (IPCC, 2014, chapitre 14,
} p. 853). 
réduire les vulnérabilités de leur territoire face aux impacts climatiques. L'adaptation suscite ainsi des attentes en matière d'innovations, politiques ou technologiques, de modifications de pratiques ou d'intégration de mesures dans les activités déjà en place (Simonet, 2011a). Dès lors, les exemples cités du secteur de l'eau et des transports laissent-ils entrevoir des opportunités économiques à l'échelle globale dans un contexte de mondialisation des échanges et des services? Parvenir à des accords signifie s'entendre, de manière officiellement établie, sur une définition s'accordant avec les points de vue et intérêts de chacun des acteurs. Cette interprétation laisse libre cours à l'imagination quant aux jeux $d$ 'influence entre les acteurs en place, que ce soient les décideurs publics lors des négociations internationales, les travaux des différents courants scientifiques ou les acteurs du secteur privé (et associatif), au moment de l'élaboration d'une terminologie facilitant l'action publique ou privée face aux changements climatiques. Doit-on conclure que dans sa réalité opérationnelle, l'adaptation aux changements climatiques est une question de négociation (Simonet et Salles, 2014) ? Les nouvelles définitions de l'adaptation reflètent-elles des résultats de négociations? Quelle est la part de l'influence des intérêts économiques dans les nouvelles terminologies de l'adaptation?

En outre, le terme "réalisables » utilisé souligne l'importance de la mise en pratique de l'adaptation, révélant que jusque-là ce point était difficilement atteint. Or, la brève analyse de cet article montre que la nouvelle terminologie témoigne du besoin de mieux cerner les contours de l'adaptation dans le contexte des changements climatiques. Cette terminologie symbolise également le reflet de la difficulté d'interprétation du concept, laquelle ne semble pas s'être estompée au fil des rapports. Ce désir de précision ne révèle-t-il pas la confusion rencontrée au stade de son opérationnalisation ? N'est-il pas le reflet d'une variabilité interprétative évoluant selon les intérêts des acteurs en présence ? Les tentatives d'affinement sémantique ne traduisent-elles pas que l'adaptation constitue une problématique en soi, voire une des principales barrières cognitives à l'action? $\mathrm{Ne}$ sont-elles pas le reflet de la complexité de penser l'adaptation dans un contexte de changements climatiques, aussi bien sur ses contours théoriques que sur l'atteinte d'une simplification favorisant sa mise en pratique? Enfin, ne confirment-elles pas que l'adaptation fait partie des concepts condamnés à évoluer au gré des contextes socioéconomiques, politiques et scientifiques? Sur ce point, Basset et Fogelman (2013) montrent bien que malgré une place proéminente et croissante dans la littérature sur les changements climatiques depuis 2007, il y a beaucoup de «déjà vu» et peu de nouveauté dans la conceptualisation de l'adaptation. Au-delà de l'adaptation, cette tendance peut également être interrogée pour les autres concepts mobilisés dans le domaine des changements climatiques : l'engouement actuel pour la résilience (Quenault, 2013), les récurrentes précisions dans les définitions de la vulnérabilité (Oppenheimer et al., 2014) ou encore l'émergence de nouvelles acceptions («Climate-Resilient Pathways») [Denton et al., 2014] représentent-elles une fuite en avant opportuniste permettant de maintenir les pratiques et les statu quo en place (Pelling, 2011) ?

Ainsi, le cinquième et dernier rapport (2014) du GIEC montre que l'adaptation demeure désormais une des préoccupations principales des changements climatiques. D'une part, du fait de l'évolution démographique et d'un mode de vie qui se généralise autour d'une consommation d'énergie non négociable issue d'hydrocarbures. Et d'autre part, parce que s'interroger sur l'adaptation à une problématique induite par les activités humaines va au-delà de la simple mise en œuvre d'une batterie de mesures pour en contrer les effets. Dès lors, comme le montre son émergence dans la sphère scientifique en tant que domaine de recherche à part entière, l'adaptation aux changements climatiques, et par extension aux changements globaux, s'inscrit dans les réflexions portant sur les modèles de développement vertueux, sur la notion de "durabilité ", mais également sur la propre capacité de l'humanité à s'adapter à ellemême (Toussaint et al., 2012).

\section{Références}

Adger, N.W., Barnett, J., 2009. Commentary: Four reasons for concern about adaptation to climate change, Environment and Planning $A, 41,2800-2805$.

Anderson, N.B., 2011. Special issue: Psychology and global climate change, American Psychologist, 66, 4, 241-328.

Basset, T.J., Fogelman, C., 2013. Déjà vu or something new?: The adaptation concept in the climate change literature, Geoforum, 48, 42-53.

Becerra, S., Peltier, A., 2009. Risques et environnement : recherches interdisciplinaires sur la vulnérabilité des sociétés, Paris, L'Harmattan.

Böhm, G., Pfister, H.-R., 2000. Action tendencies and characteristics of environmental risks, Acta Psychologica, 104, 3, 317-337.

Burton, I., Huq, S., Lim, B., Pilifosova, O., Schipper, E.L., 2002. From impacts assessments to adaptation priorities: The shaping of adaptation policy, Climate Policy, 2, 145-159.

Butzer, K.W., 1980. Adaptation to global environmental change, The Professional Geographer, 32, 3, 269-278.

Denton, F., Wilbanks, T.J., Abeysinghe, A.C., Burton, I., Gao, Q., Lemos, M.C., Masui, T., O’Brien, K.L., Warner, K., 2014. Climate-resilient pathways: Adaptation, mitigation, and sustainable development, in Field, C.B., Barros, V.R., Dokken, D.J., Mach, K.J., Mastrandrea, M.D., Bilir, T.E., Chatterjee, M., Ebi, K.L., Estrada, Y.O., Genova, R.C., 
Girma, B., Kissel, E.S., Levy, A.N., MacCracken, S., Mastrandrea, P.R., White, L.L. (Eds), Climate Change 2014: Impacts, Adaptation, and Vulnerability. Part A: Global and Sectoral Aspects, Contribution of Working Group II to the Fifth Assessment Report of the Intergovernmental Panel on Climate Change, Cambridge (UK), New York, Cambridge University Press, 1101-1131.

Eyzaguirre, J., Warren, F.J., 2014. Adaptation : établir un lien entre la recherche et la pratique, in Warren, F.J., Lemmen, D.S. (Eds), Vivre avec les changements climatiques au Canada: perspectives des secteurs relatives aux impacts et à l'adaptation, Ottawa (Ontario), Gouvernement du Canada, 253-286.

Füssel, H.-M., 2007. Adaptation planning for climate change: Concepts, assessment approaches and key lessons, Sustainability Science, 2, 265-275.

Gifford, R., 2008. Psychology's essential role in alleviating the impacts of climate change, Canadian Psychology, 49, 273-280.

Gifford, R., 2011. The dragons of inaction: Psychological barriers that limit climate change mitigation and adaptation, American Psychologist, 66, 4, 290-302.

IPCC, 1990. Climate Change 1990: The IPCC Response Strategies, Contribution of Working Group III to the Assessment Report of the Intergovernmental Panel on Climate Change, Bernthal, F. (Ed), Washington D.C., US National Science Foundation.

IPCC, 1995. Climate Change 1995: Impacts, Adaptations and Mitigation of Climate Change - Scientific-Technical Analyses, Contribution of Working Group II to the Second Assessment Report of the Intergovernmental Panel on Climate Change, Watson, R.T., Zinyowera, M.C., Moss, R.H. (Eds), Cambridge (UK), New York, Cambridge University Press.

IPCC, 2001. Climate Change 2001: Impacts, Adaptation, and Vulnerability, Contribution of Working Group II to the Third Assessment Report of the Intergovernmental Panel on Climate Change, McCarthy, J.J., Canziani, O.F., Leary, N.A., Dokken, D.J., White, K.S. (Eds), Cambridge (UK), New York, Cambridge University Press.

IPCC, 2007. Climate Change 2007: Impacts, Adaptation and Vulnerability, Contribution of Working Group II to the Fourth Assessment Report of the Intergovernmental Panel on Climate Change, Parry, M.L., Canziani, O.F., Palutikof, J.P., van der Linden, P.J., Hanson, C.E. (Eds), Cambridge (UK), New York, Cambridge University Press.

IPCC, 2014. Climate Change 2014: Impacts, Adaptation, and Vulnerability. Part A: Global and Sectoral Aspects, Contribution of Working Group II to the Fifth Assessment Report of the Intergovernmental Panel on Climate Change, Field, C.B., Barros, V.R., Dokken, D.J., Mach, K.J., Mastrandrea, M.D., Bilir, T.E., Chatterjee, M., Ebi, K.L., Estrada, Y.O., Genova, R.C., Girma, B., Kissel, E.S., Levy, A.N., MacCracken, S., Mastrandrea, P.R., White, L.L. (Eds), Cambridge (UK), New York, Cambridge University Press.

Jodha, N.S., 1989. Potential strategies for adapting to greenhouse warming: Perspectives from the developing world, in Rosenberg, N.J., Easterling, W.E., Crosson, P.R., Darmstadter, J. (Eds), Greenhouse Warming: Abatment and Adaptation, Washington D.C., Resources for the Future.
Leiserowitz, A., 2006. Climate change risk perception and policy preferences: The role of affect, imagery, and values, Climatic Change, 77, 45-72.

Marquet, V., Salles, D., 2014. L'adaptation au changement climatique en France et au Québec : constructions institutionnelles convergentes et diffusions contrastées, Critique Internationale, 1, 62, 73-91.

Mustelin, J., Kuruppu, N., Matus Kramer, A., Daron, J., de Bruin, K., Guerra Noriega, A., 2013. Climate adaptation research for the next generation, Climate and Development, 5, 3, 189-193.

Nelson, D.R., Adger, N.W., Brown, K., 2007. Adaptation to environmental change: Contributions of a resilience framework, Annual Review of Environment and Resources, 32, 395-419.

O'Brien, K., 2012. Global environmental change II: From adaptation to deliberate transformation, Progress in Human Geography, 36, 5, 667-676.

Oppenheimer, M., Campos, M., Warren, R., Birkmann, J., Luber, G., O'Neill, N., Takahashi, K., 2014. Emergent risks and key vulnerabilities, in Field, C.B., Barros, V.R. Dokken, D.J., Mach, K.J., Mastrandrea, M.D., Bilir, T.E., Chatterjee, M., Ebi, K.L., Estrada, Y.O., Genova, R.C., Girma, B., Kissel, E.S., Levy, A.N., MacCracken, S., Mastrandrea, P.R, White, L.L. (Ed.), Climate Change 2014: Impacts, Adaptation, and Vulnerability. Part A: Global and Sectoral Aspects, Contribution of Working Group II to the Fifth Assessment Report of the Intergovernmental Panel on Climate Change, Cambridge (UK), New York, Cambridge University Press, 1039-1099.

Park, S.E., Marshall, N.A., Jakku, E., Dowdd, A.M., Howden, S.M., Mendham, E., Fleming, A., 2012. Informing adaptation responses to climate change through theories of transformation, Global Environmental Change, 22, 1, 115-126.

Patt, A., 2013. Should adaptation be a distinct field of science?, Climate and Development, 5, 3, 187-188.

Pelling, M., 2011. Adaptation to Climate Change: From Resilience to Transformation, London, Routledge.

Piaget, J., 1967. Biologie et connaissance: essai sur les relations entre les régulations organiques et les processus cognitifs, Paris, Gallimard.

Quenault, B., 2013. Retour critique sur la mobilisation du concept de résilience en lien avec l'adaptation des systèmes urbains au changement climatique, EchoGéo, 24.

Quenault, B., Bertrand, F., Blond, N., Pigeon, P., 2011. Vulnérabilité et résilience au changement climatique en milieu urbain: vers des stratégies de développement urbain durable?, Projet de recherche PIRVE 20-2051, Programme interdisciplinaire ville environnement (Meddat, CNRS), Maison des sciences de l'Homme de Bretagne (MSHB).

Reser, J.P., Morrissey, S.A., Ellul, M., 2011. The threat of climate change: Psychological response, adaptation, and impacts, in Weissbecker, I. (Ed.), Climate Change and Human Well-Being: Global Challenges and Opportunities, New York, Springer Science, 19-42.

Richard, E., 2014. L'action publique territoriale à l'épreuve de l'adaptation aux changements climatiques: un nouveau 
référentiel pour penser l'aménagement du territoire? Thèse de doctorat, Université François Rabelais, Tours.

Rickards, L., Howden, S., 2012. Transformational adaptation: Agriculture and climate change, Crop and Pasture Science, 63, 240-250.

Rosenberg, N.J., Crosson, P., Easterling, W., Fredericks, K., Seejo, R., 1989. Policy options for adaptations to climate change, in United Nations Environment Programme, The Beijer Institute (Ed.), The Full Range of Responses to Anticipated Climatic Change, 69-100.

Schipper, E.L.F., 2006. Conceptual history of adaptation in the UNFCCC Process, Review of European Community $\mathcal{E}$ International Environmental Lawi, 15, 1, 82-92.

Simonet, G., 2008. Le concept d'adaptation: polysémie et implication en sciences de l'environnement. Rapport de synthèse environnementale, Université du Québec à Montréal, Montréal.

Simonet, G., 2009. Le concept d'adaptation: polysémie interdisciplinaire et implication pour les changements climatiques, Nature Sciences Société, 17, 4, 392-401.

Simonet, G., 2011a. Enjeux et dynamiques de la mise en œuvre de stratégies d'adaptation aux changements climatiques en milieu urbain, les cas de Montréal et Paris. Thèse de doctorat, Université du Québec à Montréal, Montréal.

Simonet G., 2011b. L'atelier « $\mathrm{H}$ » ou la représentation de l'adaptation dans l'élaboration du Plan Climat de Paris, VertigO, 11, 2, doi: 10.4000/vertigo.11227.

Simonet, G., 2013. Étude du Plan Stratégique de Développement Durable de la collectivité montréalaise à travers le filtre de l'adaptation aux changements climatiques, Information Géographique, 77, 3, 71-89.

Simonet, G., 2014a. L'adaptation, champ de recherche à part entière? (Avant-propos), in Adaptations aux changements environnementaux et territoires (numéro thématique sous la direction de Julien Rebotier), Sud-Ouest Européen, 37, 13-14.

Simonet, G., 2014b. Adaptation au changement climatique : la polysémie de la notion face au défi de la mise en pratique, dossier $n^{\circ} 19$ du Club ViTeCC, CDC Climat Recherche, Paris.

Simonet, G., Salles, D., 2014. Eau et changement climatique en Garonne moyenne: l'adaptation en négociation, in Adaptations aux changements environnementaux et territoires (numéro thématique sous la direction de Julien Rebotier), Sud-Ouest Européen, 37, 53-62.

Smit, B., Burton, I., Klein, R.J.T., Wandel, J., 2000. An anatomy of adaptation to climate change and variability, Climatic Change, 45, 223-251.

Toussaint, J.F., Swynghedauw, B., Boeuf, G., 2012. L'Homme peut-il s'adapter à lui-même?, Versailles, Éditions Quae.

Van Gameren, V., Weikmans, R., Zaccai, E., 2014. L'adaptation au changement climatique, Paris, La Découverte, Coll. Repères.

Vogel, C., Moser, S.C., Kasperson, R.E., Dabelko, G.D., 2007. Linking vulnerability, adaptation, and resilience science to practice: Pathways, players, and partnerships, Global Environmental Change, 17, 3, 349-364. 